This is a self-archived version of an original article. This version may differ from the original in pagination and typographic details.

Author(s): Mi, Xinru; Yang, Chungang; Chang, Zheng

Title: Multi-Resource Management for Multi-Tier Space Information Networks : A Cooperative Game

Year: 2019

Version: Accepted version (Final draft)

Copyright: @ 2019 IEEE

Rights: In Copyright

Rights url: http://rightsstatements.org/page/InC/1.0/?language=en

Please cite the original version:

Mi, X., Yang, C., \& Chang, Z. (2019). Multi-Resource Management for Multi-Tier Space Information Networks : A Cooperative Game. In IWCMC 2019 : Proceedings of the 15th International wireless communications and mobile computing conference (pp. 948-953). IEEE. International Wireless Communications and Mobile Computing Conference. https://doi.org/10.1109/iwcmc.2019.8766545 


\section{Multi-Resource Management for Multi-Tier Space Information Networks: A Cooperative Game}

\author{
Xinru Mi \\ School of Telecommunications Engineering \\ Xidian university \\ Xi' an, China \\ 552351084@qq.com
}

\author{
Chungang Yang \\ School of Telecommunications Engineering \\ Xidian university \\ Xi' an, China \\ chygang2010@163.com
}

\author{
Zheng Chang \\ University of Jyväskylä \\ Finland \\ zheng.chang@jyu.fi
}

\begin{abstract}
With the drastic increase of space information network (SIN) traffic and the diversity of network traffic types, the optimal allocation of the scarce network resources is of great significance for optimizing the SIN system capability. In this paper, we propose a multi-resource management method for multi-tier SIN using the cooperative Nash bargaining solution. Since the original problem is a non-convex problem, we firstly make logarithmic transition, and then find a tightest lower bound function to convert the initial problem into a convex one. In order to carry out the optimal bandwidth and power allocation in SIN, we construct a joint bandwidth and power allocation (JBPA) algorithm. Simulation results show the performance improvement of the JBPA scheme and the convergence of JBPA algorithm.
\end{abstract}

Index Terms-Nash bargaining solution, resource allocation, space information network, tightest lower bound function.

\section{INTRODUCTION}

With the space information network (SIN) unprecedented evolution, the limited communication capacity of traditional single satellite service model cannot meet users' requirements [1] - [3]. It is crucial to the next generation of SIN to enhance the satellites cooperation and resources sharing, which can lead to the better performance.

There are extensive research focusing on cooperative multiresource allocation problems. The authors in [4] proposed a dynamic bandwidth allocation scheme for delay-aware applications by classifying services into three levels based on the delay sensitivity. In certain cases, the resources allocated to low level services will be seized by high level services. The authors in [5] proposed a dynamic resource allocation problem to adapt diverse communicating links among satellites by jointly considering the power and bandwidth resource. To solve the allocation problem, the primal-dual method is utilized to obtain the optimal link capacity. The authors in [6] proposed a JPBA algorithm for satellite systems operating on Ka bands to meet requirements of ground users' scalability based on the observation information, i.e., weather conditions. Considering multi-task requests at the same time, an adaptive control approach is proposed based on queue bandwidth-ondemand in Geostationary Orbit (GEO) networks [7]. Besides,

This work is supported by the open research fund of National Mobile Communications Research LaboratorySoutheast UniversityNo. 2019D10; by the Fundamental Research Funds for the Central Universities(2018); by the National Science Foundation of China (91638202); in this work the authors proposed an average sum rate maximization algorithm based on cognitive radio technology, which divides satellites into the secondary users and primary users. A threshold value was defined so that the secondary users cannot interference with the primary users. However, a single resource allocation can only improve the local performance and cannot meet the overall performance of the users. Generally, power allocation should also be considered to enhance the performance of SIN when implementing bandwidth assignment.

However, the above queue-based service methods [7] can not solve the problem of concurrent users' service requests. In addition, the allocation algorithms are somewhat complicated. Thus, the game theory [8] is gradually utilized to solve the problems by investigating the competition and cooperation in communication networks, especially the Nash bargaining solution (NBS) game. For instance, the coordinated multipoint clustering scheme was formulated as a cooperative NBS problem in [9]. The authors construct the user-centric clustering problem as a cooperative NBS game, and thus ensuring fairness among users. For the sake of network efficiency, fairness and other issues, the authors in [10] proposed a bandwidth allocation framework for flexible services in centralized broadband networks based on the idea of the cooperative NBS game, resulting in total network utility maximization while thinking about fairness of users' budget. Another application, presented in [11], proposed a congestion pricing bandwidth ondemand algorithm based on game theory, which dynamically adjust the reference queue length by the significant satellite network information.

In the existing communication network system, though both mobile broadband networks and optical broadband networks have been providing various convenient services to subscriber$\mathrm{s}$, some non-trivial areas cannot be covered by these networks, i.e., space, maritime area and desert area. Therefore, Low Earth Orbit (LEO) satellites have been introduced to handle these problems, since the satellites has the advantages of short transmission delay and small path loss deriving from its short distance to ground. However, high-mobility users served by LEO satellites need continuously handover among these different satellites, which leads to the deterioration of QoS. GEO satellites can provide better coverage service to subscribers due to its wide coverage range, and thus overcome the problems 
of frequent handover. However, it is difficult for a single GEO satellite to guarantee high bandwidth demands of subscribers, such as observation tasks. Consequently, the coordinated LEO and GEO satellite system is proposed, in which LEO satellites provides subscribers with high bandwidth while GEO ones provision real-time seamless coverage to users.

TABLE I

SYSTEM PARAMETERS.

\begin{tabular}{|c|l|}
\hline System Parameter & Definition \\
\hline $\mathcal{K}$ & $\begin{array}{l}\text { a set of GEO satellite } j \\
\mathcal{K}=\{1,2, \ldots, K\}\end{array}$ \\
\hline $\mathcal{N}$ & $\begin{array}{l}\text { a set of LEO satellite } i \\
\mathcal{N}=\{1,2, \ldots, N\}\end{array}$ \\
\hline$p_{i j}$ & transmission power between satellites \\
\hline$x_{i j}$ & bandwidth assigned between satellites \\
\hline$\eta_{i, j}$ & $\begin{array}{l}\text { power spectrum density } \\
\eta_{i, j}=p_{i, j} / x_{i_{i, j}} \text { is constant }\end{array}$ \\
\hline$\gamma_{1, j}$ & bandwidth price for GEO satellite $j$ \\
\hline$\gamma_{2, j}$ & power price of GEO satellite $j$ \\
\hline
\end{tabular}

In this paper, we develop a novel joint bandwidth and power allocation (JBPA) method for multi-tier SIN using a NBS game. Specifically, three GEO satellites cooperate to serve LEO satellites to increase the total utility of LEO satellites. Based on the NBS game, a JBPA optimization framework is formulated, with the objection of maximizing the utility of LEO satellites. Due to the original problem is non-convex problem, we firstly make logarithmic transition of the variables, and then find a tightest lower bound function to convert the initial problem into a convex one. In order to implement the optimal bandwidth and power allocation in SIN, we construct a JBPA algorithm. Simulation results are verified the effectiveness and convergence of the JBPA algorithm.

The core contributions in this paper are summarized as follows:

- GEO Satellite Cooperation Framework: The JBPA framework of GEO satellite cooperation is comprehensively studied. The cooperation framework is put forward to improve the LEO satellite average sum rate and total system performance.

- Resources Allocation with NBS: The JBPA problem is formulated as a cooperation NBS game problem. This scheme achieves fairness of LEO satellites. The objective function takes the NBS fairness into consideration.

The following article is organized as follows. In Section II the system model is introduced. In Section III, we give a brief introduction to NBS, and then introduce the JBPA problem in SIN using the NBS game model. Afterwards, simulation results are shown in Section IV to certify the efficiency of the proposed JBPA algorithm. Finally, we conclude our work in Section V.

\section{System MODEL}

In this section, we describe the inter-satellite link and network model. The system utility function is defined for the following performance analysis.

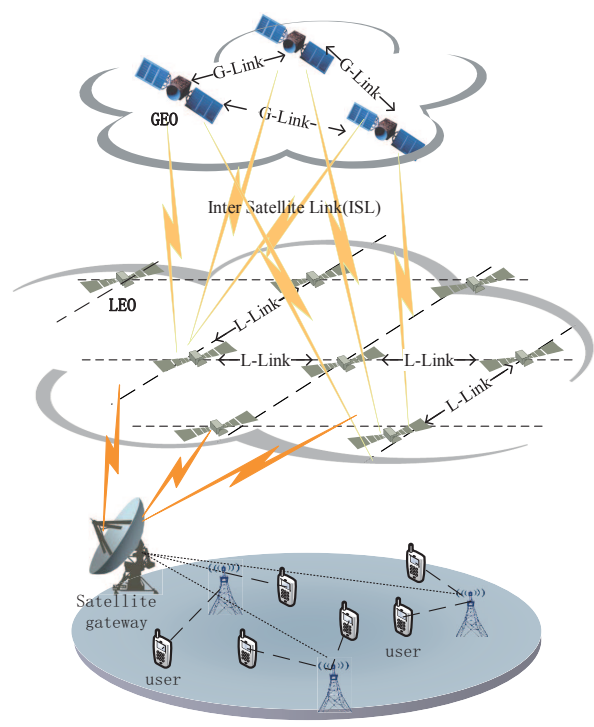

Fig. 1. Convergence analysis with different number of LEO satellite.

\section{A. Inter Satellite Link Model}

The free space loss model of inter satellite link can be noted as follows [15]

$$
g_{i, j}=\left(\frac{c}{4 \pi \cdot S_{i, j} \cdot f}\right)^{2},
$$

where, $c$ denotes the velocity of light ( $\mathrm{km} / \mathrm{s}), S_{i, j}$ is the slant range (in $\mathrm{km}$ ) between satellite $i$ and satellite $j$. The communications center frequency (in $\mathrm{Hz}$ ) of inter-satellite link is expressed as $f$.

\section{B. Network Model}

We consider a multi-tier satellite system based SIN as shown in Fig.1. The satellite system is composed of GEO, LEO satellites and ground network. GEO satellites provide wider coverage areas, and LEO satellites have advantages of shorter communication delays. With the combination, the multi-tier satellite network achieves wider area coverage via upper tier and shorter transmission delay via lower tier [12] [13]. The inter satellite link (ISL) indicates the radio communication links between the LEO satellite layer and the GEO satellite layer, while L-Link and G-Link indicate the laser communication links within LEO satellite layer and GEO satellite layer, respectively. The GEO satellite operates at the $\mathrm{Ku}$ band. In the satellite network, three GEO satellites cooperate to serve the set of LEO satellites through interactive information. We used orthogonal frequency division multiple access (OFDMA) [14] systems with different LEO satellites share frequency band and time resources and interference from LEO satellite layer can be ignored via satellite coordination in the downlink. The parameters about the satellite network are shown in Table I.

Let $r_{i, j}\left(x_{i, j}, p_{i, j}\right)$ be a link reward function between LEO satellite layer and GEO satellite layer. The link reward function can be presented as 


$$
r_{i, j}\left(x_{i, j}, p_{i, j}\right)=x_{i, j} \log _{2}\left(1+g_{i, j} p_{i, j} / x_{i, j}\right),
$$

where $x_{i, j}$ and $p_{i, j}$ represent the allocated bandwidth and power to LEO satellite $i . p_{i, j} / x_{i, j}=\eta_{i, j}$ is power spectrum density, which is a constant. The link reward function reflects the improvement performance of the LEO satellite by the ISL.

The bandwidth and power price function $c_{i, j}\left(x_{i, j}, p_{i, j}\right)$ can be denoted as

$$
c_{i, j}\left(x_{i, j}, p_{i, j}\right)=\gamma_{1, j} x_{i, j}+\gamma_{2, j} p_{i, j},
$$

where $\gamma_{1, j}$ and $\gamma_{2, j}$ are used to represent bandwidth and power price for GEO satellite $j$, According to system efficiencies, different GEO satellites charge different prices to LEO satellites. Moreover, we combine link reward function and cost function to obtain the link utility function $u_{i, j}\left(x_{i, j}, p_{i, j}\right)$ for LEO satellites as follows:

$$
\begin{aligned}
& u_{i, j}\left(x_{i, j}, p_{i, j}\right)=r\left(x_{i, j}, p_{i, j}\right)-c\left(x_{i, j}, p_{i, j}\right) \\
= & x_{i, j} \log _{2}\left(1+g_{i, j} p_{i, j} / x_{i, j}\right)-\gamma_{1, j} x_{i, j}-\gamma_{2, j} p_{i, j} .
\end{aligned}
$$

With more usage of power and bandwidth resources, the higher link reward, the higher payoff of LEO satellites for occupying more resource. Therefore, link reward and resource cost need to be balanced by using a cooperative bargaining game.

\section{A CoOperative Bargaining GAME MOdel}

In order to clarify the NBS, we first make a brief introduction to NBS. Furthermore, we introduce the JBPA in the SIN using the NBS game model.

\section{A. Basics of NBS}

Game theory is a significant mathematical branch and has been extensively researched. In the perspective of maximization of the collective revenue or its own profits, the game theory can be divided into the cooperative game and non-cooperative game. The NBS can be described as cooperative game theory [16] - [18]. Since the satellite resources by GEO satellite are limited, there is a competitive relationship between LEO satellites. Therefore, the LEO satellite competing resources for GEO satellite can be formulated as a bargaining problem. Let LEO satellite group $N=\{1,2, \ldots, N\}$ represent the players and $u_{i, j}^{\min }$ represent the minimal payoff of each LEO satellite $i$ for GEO satellite $j$. Let $U=\left(u_{1}, u_{2}, \ldots, u_{i}^{\min }, \ldots, u_{N}\right)$ represent the set of possible payoff allocation when the LEO satellites work together and $u=\left\{u_{i, j} \mid u_{i, j} \geq u_{i, j}^{\min }, \forall i \in N\right\}$ be a nonempty bounded set. Furthermore, we denote $u_{i, j}^{\min }=$ $\left(u_{1,1}^{\min }, \ldots, u_{N, 1}^{\min }, u_{1,2}^{\min }, \ldots, u_{N, 2}^{\min }, \ldots, u_{i, j}^{\min }, \ldots, u_{N, K}^{\min }\right)$, and then the pair $\left(u_{i, j}, u_{i, j}^{\min }\right)$ can be defined as the $N$-participator bargaining problem. They may have an unlimited amount of Pareto optimal points. In consequence, we should to choose the bargaining result through NBS fairness criteria. A unique and fair Pareto optimal point is provided by NBS and explained as follows.

Definition 1: $u_{\text {opt }}$ is said to be an NBS in $u_{i, j}$ for $u_{i, j}^{\min }$, $u_{o p t}=\phi\left(u_{i, j}, u_{i, j}^{\min }\right)$, if the following conditions are satisfied.
1) Individual Rationality: $\bar{u}_{i}=\sum_{i=1}^{N} \bar{u}_{i, j} \geq u_{i, j}^{\min }, \forall i$.

2) Feasibility: $u_{o p t} \in U$.

3) Pareto Optimality: $u_{o p t}$ is a Pareto optimal.

4) Independence of Independent Substitutes: If $u_{o p t} \in$ $u_{i, j}^{\prime} \subset u_{i, j}, u_{\text {opt }}=\phi\left(u_{i, j}, u_{i, j}^{\min }\right)$, then $u_{\text {opt }}=\phi\left(u_{i, j}^{\prime}, u_{i, j}^{\min }\right)$.

5) Independence of Linear Transformations: For any linear transformation $f$, if the problem $\phi\left(u_{i, j}, u_{i, j}^{\min }\right)$ is $u_{o p t}$, then the problem $f\left(\phi\left(u_{i, j}, u_{i, j}^{\min }\right)\right)$ is $f\left(u_{\text {opt }}\right)$.

6) Symmetry: If $U$ is invariant under all exchanges of players, $\phi_{i, j}\left(U, u_{i, j}^{\min }\right)=\phi_{i^{\prime}, j}\left(U, u_{i, j}^{\min }\right), \forall i, i^{\prime} \in N$.

The uniqueness and existence of NBS can be described in theorem 1 .

Theorem1: Uniqueness and Existence of NBS: There is a unique and fair solution for $\phi\left(u_{i, j}, u_{i, j}^{\min }\right)$ that satisfies above six conditions in Definition 1 which can be followed as [18]:

$$
\phi\left(u_{i, j}, u_{i, j}^{\min }\right) \in \arg \underset{u \in U, u_{i, j} \geq u_{i, j}^{\min } \forall i}{\max } \prod_{i=1}^{N}\left(u_{i, j}-u_{i, j}^{\min }\right) .
$$

\section{B. Game Model and Problem Formulation}

Through the above introduction, combined with (3), the JBPA in SIN using NBS can be expressed as follows:

$$
\begin{aligned}
& \max _{x, p} \prod_{i=1}^{N}\left(u_{i, j}-u_{i, j}^{\min }\right) \\
& \mathrm{C} 1: \sum_{j=1}^{\hat{K}} x_{i, j} \log _{2}\left(1+g_{i, j} \eta_{i, j}\right) \geq \text { rate }_{i}, \forall j \in \mathcal{K} \\
& \mathrm{C} 2: \sum_{j=1}^{\hat{K}} x_{i, j}>0, \forall j \in \mathcal{K} \\
& \mathrm{C} 3: \sum_{j=1}^{\hat{K}} p_{i, j}>0, \forall j \in \mathcal{K} \\
& \mathrm{C} 4: \sum_{i=1}^{N} x_{i, j} \leq X_{j}^{\max }, \forall j \in \mathcal{K} \\
& \mathrm{C} 5: \sum_{i=1}^{N} p_{i, j} \leq P_{j}^{\max }, \forall j \in \mathcal{K} .
\end{aligned}
$$

Let $u_{i, j}^{\min }=0$ to ensure the proportion of fair. Constraint C1 implies that bandwidth needs to meet the minimum LEO satellite rate requirement rate $_{i}$, and we have $x_{i, j}^{m, r e q}=$ $\frac{\text { rate }_{j, m}}{\sum_{j=1}^{\hat{K}} \log _{2}\left(1+g_{i, j} p_{i, j}^{m} / x_{i, j}^{m}\right)}$. Constraints C2 and C3 are ensure the non-negativity of the allocated bandwidth and power, respectively. Constraint $\mathrm{C} 4$ ensures the bandwidth allocation to all LEO satellites should be no more than the total available power denoted by $X_{j}^{\max }$; Constraint $\mathrm{C} 2$ is the same as $\mathrm{C} 1$. Take the logarithm transformation of the main function in (5), the NBS game problem can be transformed as

$$
\max _{x, p} \sum_{i=1}^{N} \ln \left(u_{i, j}\right)
$$




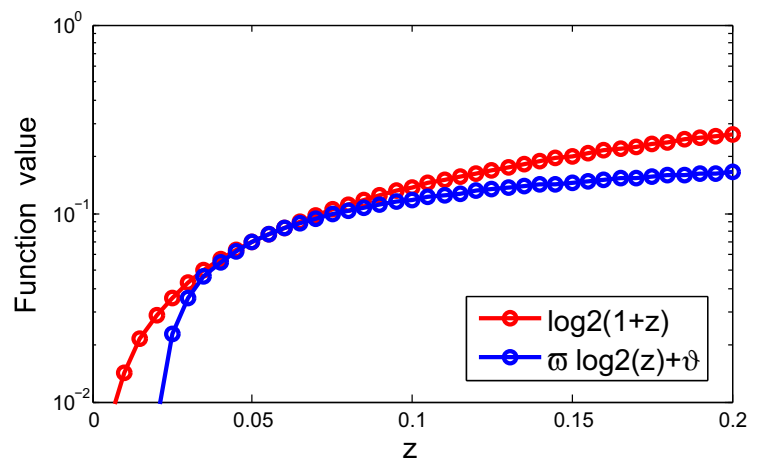

Fig. 2. The function $\log _{2}(1+z)$ and its tightest lower function $\varpi \log _{2} z+\vartheta$ with $z_{0}=0.05$

In (8), we can see the $\ln \left(\frac{1}{\gamma_{2, j} p_{i, j}^{m}}\right)$ and $\ln \left(\frac{1}{\gamma_{1, j}}\right)$ are convex functions. It can be followed that (6) is a convex problem by verifying that (9) is a convex problem.

$$
u^{\prime}\left(x_{i, j}, p_{i, j}\right)=\ln \left(1+g_{i, j} \frac{p_{i, j}}{x_{i, j}}\right) .
$$

Note that, $u^{\prime}\left(x_{i, j}, p_{i, j}\right)$ is not a convex problem [19] but is a classic d.c. (difference of two concave functions) structure. Generally, it is difficult to obtain the global optimal solutions for optimization problems with the d.c. structure. However, these problems can be converted to strictly convex problems [20] following Lemma1.

Lemma1: For any $z \geq 0$, the following bound is derived as

$$
\varpi \log z+\vartheta \leq \log (1+z)
$$

where the lower bounds coefficients $\varpi$ and $\vartheta$ are given as

$$
\left\{\begin{array}{c}
\varpi=\frac{z_{0}}{1+z_{0}}, \\
\vartheta=\log _{2}\left(1+z_{0}\right)-\frac{z_{0}}{1+z_{0}} \log _{2} z_{0},
\end{array}\right.
$$

where, $z_{0}$ are arbitrary nonnegative numbers. As shown in Fig.2, we can seen that the lower bound function $\varpi \log z+\vartheta \leq$ $\log (1+z)$ is tight at $z=z_{0}$.

According to lemma 1, (9) can be relaxed as

$$
\begin{array}{r}
u^{\prime}\left(x_{i, j}, p_{i, j}\right)=\ln \left(1+g_{i, j} \frac{p_{i, j}}{x_{i, j}}\right) \\
\geq \varpi_{i, j} \ln \left(g_{i, j} \frac{p_{i, j}}{x_{i, j}}\right)+\vartheta_{i, j} .
\end{array}
$$

From (12) it can be seen that $u^{\prime}\left(x_{i, j}, p_{i, j}\right)$ is still a nonconvex problem. Applying $\tilde{p}_{i, j}=\ln p_{i, j}$ and $\tilde{x}_{i, j}=\ln x_{i, j}$, we can convert $u^{\prime}\left(x_{i, j}, p_{i, j}\right)$ to

$$
\begin{array}{r}
u^{\prime}\left(x_{i, j}, p_{i, j}\right) \geq \varpi_{i, j} \ln \left(g_{i, j} \frac{p_{i, j}}{x_{i, j}}\right)+\vartheta_{i, j}, \\
=\varpi_{i, j} \ln \left(\frac{g_{i, j} e^{\tilde{p}_{i, j}}}{e^{\tilde{x}_{i, j}}}\right)+\vartheta_{i, j}, \\
=\varpi_{i, j}\left[\ln \left(g_{i, j}\right)+\tilde{p}_{i, j}-\tilde{x}_{i, j}\right]+\vartheta_{i, j} .
\end{array}
$$

Since $\ln x$ is a concave function and others are the linear operation and concave terms. As we all known, sum function and exp function is convex problem.

Finally, the optimization problem in (6) is transformed into a convex problem as

$$
\begin{array}{ll}
\max _{\tilde{x}, \tilde{p}} & \sum_{i=1}^{N} \ln \left(\tilde{u}_{i, j}\right) \\
\mathrm{C} 1: & \sum_{j=1}^{\hat{K}} \tilde{x}_{i, j} \log _{2}\left(1+g_{i, j} \tilde{p}_{i, j} / \tilde{x}_{i, j}\right) \geq \text { rate }_{i}, \\
\mathrm{C} 2: \quad & \sum_{j=1}^{\hat{K}} \tilde{x}_{i, j}>0, \forall i, j, \\
\mathrm{C} 3: & \sum_{j=1}^{\hat{K}} \tilde{p}_{i, j}>0, \forall i, j, \\
\mathrm{C} 4: & \sum_{i=1}^{N} \tilde{x}_{i, j} \leq X_{j}^{\max }, \forall j, \\
\mathrm{C} 5: & \sum_{i=1}^{N} \tilde{p}_{i, j} \leq P_{j}^{\max }, \forall j .
\end{array}
$$

Through the above analysis, it can be seen that the expression of $\tilde{u}_{i, j}$ is sum of some linear and concave terms, and is strictly convex problem in $\left(\tilde{x}_{i, j}, \tilde{p}_{i, j}\right)$. Furthermore, the constrains $\mathrm{C} 1$ is convex. Thus, (14) is a convex optimization problem.

In order to solve optimization (14), we propose the JBPA algorithm to obtain the lower bound coefficients. First, initializes iteration counter $c=0$, lower bound coefficients $\varpi=0$ and $\vartheta=0$, the maximum number of iteration counter is $C_{\max }$. Second, obtaining $\tilde{p}_{i, j}$ and $\tilde{x}_{i, j}$ by solving the optimization problem in (14). Thus, power $p_{i, j}$ and bandwidth $x_{i, j}$ allocation to LEO satellite $i$ by the GEO satellite $j$ are

$$
\begin{aligned}
& \ln \left(u_{i, j}\right)=\ln \left(r\left(x_{i, j}, p_{i, j}\right)-c\left(x_{i, j}, p_{i, j}\right)\right)=\ln \left(x_{i, j} \log _{2}\left(1+g_{i, j} p_{i, j} / x_{i, j}\right)-\gamma_{1, j} x_{i, j}-\gamma_{2, j} p_{i, j}\right) \\
& =\ln \left(\frac{\log _{2}\left(1+g_{i, j} p_{i, j} / x_{i, j}\right)}{\gamma_{1, j}} \cdot \frac{1}{\gamma_{2, j} p_{i, j}}\right)=\ln \left(\frac{\log _{2}\left(1+g_{i, j} p_{i, j} / x_{i, j}\right)}{\gamma_{1, j}}\right)+\ln \left(\frac{1}{\gamma_{2, j} p_{i, j}}\right) \\
& =\ln \left(\frac{1}{\gamma_{1, j}}\right)+\ln \left(\frac{\ln \left(1+g_{i, j} \frac{p_{i, j}}{x_{i, j}}\right)}{\ln 2}\right)+\ln \left(\frac{1}{\gamma_{2, j} p_{i, j}}\right)
\end{aligned}
$$


obtained. Finally, the iteration continues until convergence is reached or iteration counter $c=C_{\max }$.

For specific $\varpi$ and $\vartheta$, we can solve problem (14) with gradient descent method for the dual problem of (14), which is formulated as

$$
\begin{aligned}
&(14)-\text { Dual } \min _{\xi, \gamma, \delta, \sigma, \kappa}\{R(u)\} \\
& \text { s.t. } \xi \succ 0, \gamma \succ 0, \delta \succ 0, \sigma \succ 0, \kappa \succ 0 .
\end{aligned}
$$

where $R(u)$ is the Lagrangian dual function, in which $R(u)$ can be written as

$$
\begin{aligned}
& R(u)=\max _{\tilde{x}, \tilde{p}} \sum_{i=1}^{N} \ln \left(\tilde{u}_{i, j}^{m}\right) \\
& +\sum_{i=1}^{N} \xi_{i}\left(\text { rate }_{j, m}-\sum_{j=1}^{\hat{K}} \tilde{x}_{i, j}^{m} \log _{2}\left(1+g_{i, j} \tilde{p}_{i, j}^{m} / \tilde{x}_{i, j}^{m}\right)\right) \\
& +\sum_{i=1}^{N} \gamma_{i}\left(\sum_{i=1}^{N} \tilde{x}_{i, j}^{m}-X_{j}^{\max }\right)+\sum_{i=1}^{N} \delta_{i}\left(\sum_{i=1}^{N} \tilde{p}_{i, j}^{m}-P_{j}^{\max }\right) \\
& -\sum_{i=1}^{N} \sigma_{i} \tilde{x}_{i, j}^{m}-\sum_{i=1}^{N} \kappa_{i} \tilde{p}_{i, j}^{m} .
\end{aligned}
$$

The variable $\xi_{i}, \gamma_{i}, \delta_{i}, \sigma_{i}, \kappa_{i}$ are the Lagrangian multipliers. Then the optimal solutions can be derived with the KarushKuhnTucker (KKT) conditions.

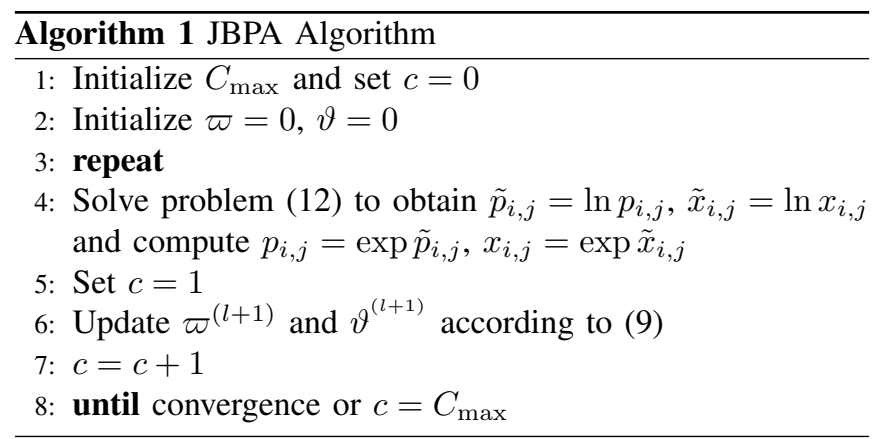

\section{Convergence Analysis}

The prerequisite of obtaining the optimal solutions is the lower bound function. Since the acquisition of the lower bound is the prerequisite for the optimal solutions, we give the following proposition to investigate the convergence of JPBA Algorithm.

Proposition 1: The JPBA algorithm monotonically improves the value of the objective function at each iteration and finally converges.

Proof: Let $x_{l}$ and $p_{l}$ be the optimized value after $l$ iterations. Then, we have

$$
\begin{aligned}
\ldots & \stackrel{(d)}{\leq} u\left(x_{l}, p_{l}\right) \stackrel{(a)}{=} \tilde{u}_{\left(\varpi_{l}, \vartheta_{l}\right)}\left(x_{l}, p_{l}\right) \stackrel{(b)}{\leq} \tilde{u}_{\left(\varpi_{l}, \vartheta_{l}\right)}\left(x_{l+1}, p_{l+1}\right) \\
& \stackrel{(d)}{\leq} \tilde{u}_{\left(x_{l+1}, p_{l+1}\right)} \stackrel{(a)}{=} \tilde{u}_{\left(\varpi_{l+1}, \vartheta_{l+1}\right)}\left(x_{l+1}, p_{l+1}\right) \stackrel{(b)}{\leq} \ldots
\end{aligned}
$$

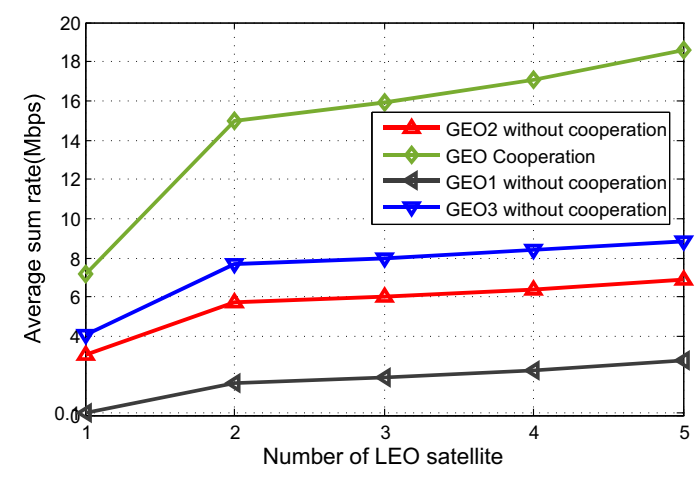

Fig. 3. Average sum rate for the GEO satellites cooperation and noncooperation.

where, (a) holds because that the relaxation is tight at the current $g_{i, j} p_{i, j}^{m} / x_{i, j}^{m}$ values; (b) is due to optimization problem (14) is a strictly concave problem; (d) holds is due to (13). Most importantly, for the fixed total transmitting power and the channel gains, the optimal sum rate is bounded, and the procedure must converge.

\section{Simulation Results}

\section{A. Simulation Environment}

Our simulation scenario composes of three GEO satellites and fifteen LEO satellites from the Satellite Tool Kit (STK). Wherein, three GEO satellites lie at nominal longitudes of $76.95^{\circ} \mathrm{E}, 176.76^{\circ} \mathrm{E}$ and $16.65^{\circ} \mathrm{E}$, respectively and each GEO satellite can serve no more than five LEO satellites simultaneously. Fifteen LEO satellites are uniformly distributed three sun-synchronous polar orbits at a height of $778 \mathrm{~km}$, with inclination of $98.5^{\circ}$. The planning period of the scenario is one day (5 Jun 2018 3:00-6 Jun 2018 3:00), and the time interval is 5 minutes. In addition, the bandwidth of three GEO satellites are $8 \mathrm{MHz}, 7 \mathrm{MHz}$, and $9 \mathrm{MHz}$, respectively. Fifteen LEO satellites are arbitrarily placed in the overlapping coverage area of three GEO satellites. The maximum transmission power of GEO satellites are 20w, 22w and 26w, respectively. Due to the different system efficiencies of three GEO satellites, we set the power price and bandwidth price parameters as $\gamma_{1, j}=[0.8,0.8,1.0]$ and $\gamma_{2, j}=[0.8,1.0,0.9]$ [21].

\section{B. Simulation Results}

Fig.3 depicts the average sum rate of the GEO satellite cooperation scheme and noncooperation scheme with varying LEO satellites quantity. It is obviously seen that the average sum rate rises for all cases as the number of satellites changes. Furthermore, the effective receiving power $p_{i, j}$ will be enlarged owing to the increasing amount of LEO satellites. And utility $u_{i, j}$ increases along with $p_{i, j}$. In addition, penalty term $\gamma_{2, j} p_{i, j}$ will effectively prevent the increase of utility, which perfectly demonstrating a NBS game process.

Fig.4 investigates the convergence of the JBPA algorithm with different numbers of LEO satellites. It is observed that the 


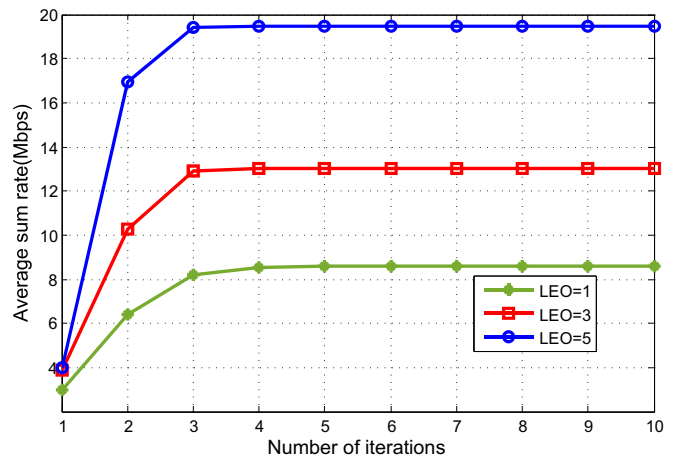

Fig. 4. Convergence analysis with different number of LEO satellite.

JBPA algorithm converges to the stable solution monotonically within six iterations.

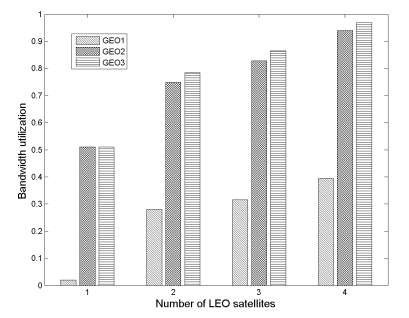

(a) Bandwidth resource utilization

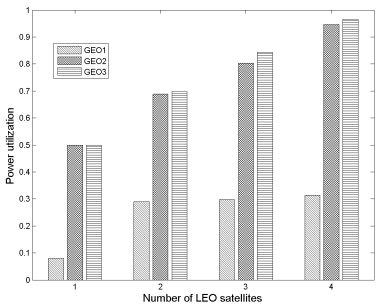

(b) Power resource utilization

Fig. 5. Resource utilization

For the sake of verify the performance of GEO satellite scheme, we research the utilization of bandwidth and power resources, as shown in Fig.5. Besides, with the quantity of LEO satellites increases, the utilization function $u_{i, j}$ gradually increases. Finally, the resources utilization achieves the highest if there are four LEO satellites. The conclusions verify that GEO satellite cooperative allocation can make GEO satellite system achieve the highest resource utilization.

\section{CONCLusion}

In this paper, we proposed multi-resource management for multi-tier SIN using a cooperative bargaining game. Then we solved this non-convex problem by effective transformation method as well as the logarithmic transformation of the variables to transform the prime problem into a convex optimization problem, and then deduced an best solution by the proposed iteration algorithm. Numerical results show that with the proposed JPBA algorithm, the system resource utilization and the average sum rate can be improved by $40 \%$ and $10 \%$, respectively.

\section{REFERENCES}

[1] Y. Kawamoto, H. Nishiyama, N. Kato, and N. Kadowaki, "A Traffic Distribution Technique to Minimize Packet Delivery Delay in Multilayered Satellite Networks," IEEE Transactions on Vehicular Technology, vol. 62, no. 7, pp. 3315-3324, Sep. 2013.
[2] H. Nishiyama, Y. Tada, N. Kato, N. Yoshimura, M. Toyoshima, and N. Kadowaki, "Toward Optimized Traffic Distribution for Efficient Network Capacity Utilization in Two-Layered Satellite Networks," IEEE Transactions on Vehicular Technology, vol. 62, no. 3, pp. 1303-1313, Mar. 2013.

[3] Y. Wang, W. Zhuang, S. Zhang, N. Zhang, R. Liu, and J. Li , "MultiResource Coordinate Scheduling for Earth Observation in Space Information Networks," IEEE Journal on Selected Areas in Communications, vol. 36, no. 2, pp. 268-279, Feb. 2018.

[4] C. Wu, W. Sun, W. Yu, H. Lu, Z. Song, and Y. Ma, "DDBA: A Method of Dynamic Deprivable Bandwidth Allocation for Space Information Network," 2009 Fifth International Conference on Mobile Ad-hoc and Sensor Networks, Fujian, China, pp. 371-375, Dec. 2009.

[5] Z. Ji, Y. Wang, W. Feng, and J. Lu,"Delay-Aware Power and Bandwidth Allocation for Multiuser Satellite Downlinks," IEEE Communications Letters, vol. 18, no. 11, pp. 1951-1954, Nov. 2014.

[6] S. Mukherjee, E Cianca, and M. D Sanctis, "Multidimensional resource management algorithm for a multibeam EHF satellite system," 2014 IEEE Aerospace Conference, Big Sky, MT, Mar.2014.

[7] L. Pimpinella, A. Fiaschetti, and A. Pietrabissa, "Dynamic bandwidth allocation in satellite networks," European Control Conference (ECC), Budapest, Hungarian, Aug. 2009, pp. 2948-2953.

[8] Z Han , D Niyato, W Saad ,and T Basar, Game theory in wireless and communication networks: theory, models, and applications, Cambridge university press, 2012.

[9] M. M. Abdelhakam, M. M. Elmesalawy, K. R. Mahmoud, and I. I. Ibrahim, "A Cooperation Strategy Based on Bargaining Game for Fair User-Centric Clustering in Cloud-RAN," IEEE Communications Letters, vol. 22 , no. 7, pp. 1-1, Apr. 2018.

[10] H. Yaiche, R. R. Mazumdar, and C. Rosenberg, "A game theoretic framework for bandwidth allocation and pricing in broadband networks," IEEE/ACM Transactions on Networking, vol. 8, no. 5, pp. 667-678, Oct. 2000.

[11] A. Fiaschetti, A. Pietrabissa, M. Fiaschetti, and M. Petrone, "Congestion pricing for dynamic bandwidth allocation in satellite networks: A gametheoretic approach," IEEE First AESS European Conference on Satellite Telecommunications (ESTEL), Rome, Italy, Oct. 2012.

[12] F. Vatalaro, G. E. Corazza, C. Caini, and C. Ferrarelli, "Analysis of LEO, MEO, and GEO global mobile satellite systems in the presence of interference and fading," IEEE Journal on Selected Areas in Communications, vol. 13, no. 2, pp. 291-300, Feb. 1995.

[13] M. Sheng, Y. Wang, J. Li, R. Liu, D. Zhou, and L. He, "Toward a Flexible and Reconfigurable Broadband Satellite Network: Resource Management Architecture and Strategies," IEEE Wireless Communications, vol. 24, no. 4, pp. 127-133, Aug. 2017.

[14] Series S. Multi-carrier based transmission techniques for satellite systems, Electronic Publication, 2011.

[15] D. Zhou, M. Sheng, R. Liu, Y. Wang, and J. Li, “Channel-Aware Mission Scheduling in Broadband Data Relay Satellite Networks," IEEE Journal on Selected Areas in Communications, vol. 36, no. 4, pp. 1-13, May. 2018

[16] H. Yaiche, R. R. Mazumdar, and C. Rosenberg, "A game theoretic framework for bandwidth allocation and pricing in broadband networks," IEEE/ACM Transactions on Networking, vol. 8, no. 5, pp. 667-678, Oct. 2000.

[17] D. Grosu, A. T. Chronopoulos, and M. Ying , "Load balancing in distributed systems: an approach using cooperative games," Proceedings 16th International Parallel and Distributed Processing Symposium, Ft. Lauderdale, FL, pp. 52-61, Apr. 2001.

[18] R. B. Myerson . Game theory, Harvard university press, 2013.

[19] H. Li, and K. Wang, "Weighted Bandwidth-Power Product Optimization in Downlink Femtocell Networks," IEEE Communications Letters, vol. 19, no. 9, pp. 1588-1591, Sept. 2015

[20] J. Papandriopoulos, S. Dey, and J. Evans, “Optimal and Distributed Protocols for Cross-Layer Design of Physical and Transport Layers in MANETs," IEEE/ACM Transactions on Networking, vol. 16, no. 6, pp. 1392-1405, Dec. 2008.

[21] R. Wu, J. Gao, G. Xie, Y. Liu, M. Guo, and S. Liu, "QoE-aware bandwidth allocation method with GEO satellites cooperation in distributed constellation network," International Conference on Wireless Communications \& Signal Processing (WCSP), Nanjing, China, Oct. 2015. 1-5. 